Canad. Math. Bull. Vol. 25 (2), 1982

\title{
A GROUP THEORETIC APPROACH TO THE EQUATIONS OF PLASMA PHYSICS
}

\author{
JERROLD E. MARSDEN*
}

\begin{abstract}
This paper concerns the interaction between group theory and classical mechanies in general and with the application of this theory to plasma physies in particular.

Mechanies has had a profound role in the historical development of mathematics. Leading original thinkers in pure mathematics such as Newton, Euler, Lagrange, Jacobi, Laplace, Cauchy, Gauss, Riemann, Poincaré, Hilbert, Birkhoff, Smale and Arnold were also great original thinkers in various facets of mechanics. The interaction between mechanies and pure mathematics remains one of the most active and flourishing areas of current research. It is this flavor which I hope to convey in this lecture.
\end{abstract}

Ackmowledgements. Much of the work that I will describe below was done in collaboration with Alan Weinstein (see Marsden and Weinstein [1981]). A seminal paper of Morrison [1980] originally shown to us by Allan Kaufman was our starting point. Our ideas were developed in a series of lectures given by both of us to Kaufman's plasma physics-dynamics seminar early in 1981 at Berkeley. The participant's enthusiasm and encouragement were very important.

I thank the officers of the Canadian Mathematical Society for the opportunity and honor to present these ideas in the Jeffrey-Williams lecture.

I wish to dedicate this lecture to my undergraduate teachers from Toronto who had such a positive influence on my career, especially Professors Atkinson, Coxeter, Davis, Duff, Pillow, Rooney, Rund, Scherk, Scherk and Vanstone. It was through them that I first appreciated the fact that pure and applied mathematics have a magnificant intersection within mathematics.

\$1. Introduction. Commencing around 1750 , Euler discovered and investigated the basic equations governing two of the most important mechanical systems, namely the rigid body and a perfect incompressible fluid. He would have been pleased with the fundamental paper of Arnold [1966] which showed

Received by the editors September $20,1981$.

* This paper is based on the Jeffrey-Williams lecture given at the meeting of the Canadian Mathematical Society, Dalhousie University, Halifax, Nova Scotia, May 28, 1981 upon the invitation of the Research Committee of the CMS. Research partially supported by NSF grant MCS-78-06718, ARO contract DAAG-29-79-C-0086, and the Miller Institute for Basic Research.

AMS (1980) Classification: 58D, 58E, 58F, 70H, 70W. 
that both of these sets of equations are of a special Hamiltonian type which is associated with a Lie group; in fact the group may be regarded as the configuration space of the system. For the rigid body the group is $S O(3)$, the proper orthogonal transformations of Euclidean 3-space $\mathbb{R}^{3}$ and for a perfect incompressible fluid, the group is $\mathscr{D}_{\text {vol }}$, the group of volume preserving diffeomorphisms of a region in $\mathbb{R}^{2}$ or $\mathbb{R}^{3}$.

The paper of Ebin and Marsden [1970] showed how to put Arnold's ideas for a perfect fluid into a rigorous infinite dimensional context and showed that this procedure leads to new existence, and convergence theorems that are ultimately useful in numerical work (see Chorin et. al. [1978] and references therein). It was noted there that many of the results on $\mathscr{D}_{\text {vol }}$ extend to $\mathscr{P}$, the group of canonical transformations of a given symplectic manifold i.e. a given phase space.

Here we show that there is a fundamental system associated to $\mathscr{S}$ in the same way that the rigid body is associated with $S O(3)$ and fuids are associated with $\mathscr{D}_{\text {vol }}$. This system consists of the Poisson-Vlasov equations for a collisionless plasma and its generalization, the Maxwell-Vlasov equation. These equations are as fundamental to the plasma community as the Navier-Stokes equations are to the fluids community.

We list below some of the important mechanical systems that have as configuration spaces a Lie group; (some additional details are provided in the next section).

System

1. free rigid body

2. heavy top

3. perfect incompressible fluid

4. compressible fluid

5. Korteweg de Vries Equation

6. Toda lattice

7. Liouville equation of any Hamiltonian system

8. Heisenberg equation of quantum mechanics

9. Lax equations for non-linear waves

10. Poisson-Vlasov equations
Group

$S O(3)=$ rotation group

$E(3)=$ Euclidean group

$\mathscr{D}_{\text {vol }}=$ volume preserving diffeomorphisms

$\mathscr{D} \times \mathscr{F}=$ semi-direct product of diffeomorphisms and functions

$\mathscr{I} \mathscr{F}=$ group of invertible Fourier Integral operators

$H=$ invertible lower triangular matrices

$\mathscr{S}=$ canonical transformations

$\mathscr{U}(\mathscr{H})$, unitary group of complex Hilbert space

$u=$ unitary group

$\mathscr{S}=$ group of canonical transformations

There are other basic systems in mechanics whose connection with a Lie 
group is less direct. For these, there is a more general way to link Lie groups and mechanical systems via symmetry and reduction (Marsden and Weinstein [1974]). This procedure includes many additional interesting systems besides those already listed. A few are:

System

11. Maxwell's equations

12. Yang-Mill's equations

13. Einstein's equation of General Relativity

14. Supergravity

\section{Group}

$\mathscr{G}=$ gauge group of electrodynamics

$\mathscr{G}=$ automorphisms of a principle bundle

$\mathscr{D}=$ diffeomorphism group of spacetime

$S$ = group of supersymmetry transformations.

In the following section we shall explain the coadjoint orbit scheme which encompasses systems 1-10 and shall indicate how the Euler equations for a rigid body in system 1 and how systems 8 and 9 fit this scheme. Then we go on to explain how Maxwell's equations can be described in terms of reduction. Finally we describe system 10 and how it is coupled with Maxwell's equations to produce the Maxwell-Vlasov system.

It will be necessary to assume some background in modern mechanics and Lie groups. See for example Arnold [1978] or Abraham and Marsden [1978].

\$2. Coadjoint orbit structures. Let $G$ be a Lie group and $(B)$ its Lie algebra. Let $\mathcal{G}^{*}$ be the dual space of $\mathcal{G}$. Let $\mu \in \mathcal{F}^{*}$ denote a typical point. Let $F: \mathbb{G}^{*} \rightarrow \mathbb{R}$ be a smooth real-valued function defined on $\mathbb{S}^{*}$. Define its functional derivative $\delta F / \delta \mu: \mathbb{S}^{*} \rightarrow$ (S) by

$$
D F(\mu) \cdot \nu=\left\langle\nu, \frac{\delta F}{\delta \mu}\right\rangle
$$

where $\nu$ is an arbitrary element of $\mathbb{H}^{*}, D F(\mu): \mathbb{H}^{*} \rightarrow \mathbb{R}$ is the (Fréchet) derivative of $f$ at $\mu,\langle$,$) is the pairing between \mathcal{G}^{*}$ and $\mathcal{G}$ and $\delta F / \delta \mu$ is understood to be evaluated at $\mu$.

Define a bracket on functions $F, G$ by

$$
\{\{F, G\}\}(\mu)=-\left\langle\mu,\left[\frac{\delta F}{\delta \mu}, \frac{\delta G}{\delta \mu}\right]\right\rangle
$$

where [,] denotes the Lie bracket of the Lie algebra $(\mathbb{S}$.

We call $\{\{\}$,$\} the KAKS bracket after Kirillov, A rnold, Kostant and Souriau$ who used it in various forms in their work in representation theory, Hamiltonian systems on Lie groups and quantization.

The KAKS bracket is clearly antisymmetric, is bilinear in $F$ and $G$ and is a derivation in $F$ and $G$. Somewhat less obvious, but true, is Jacobi's identity. Thus, the KAKS bracket makes $C^{\infty}\left(\mathbb{G}^{*}, \mathbb{R}\right)$, the $C^{\infty}$ real valued functions on $\mathbb{B}^{*}$ 
into a Lie algebra. One says that $\mathcal{B}^{*}$ together with $\{\{\}$,$\} forms a Poisson$ manifold; c.f. Guillemin and Sternberg [1980].

If $H: \mathfrak{G}^{*} \rightarrow \mathbb{R}$ is a given (energy) function there is a unique vector field $X_{H}$ on $\left(^{*}\right.$ such that for any $F \in C^{\infty}\left(G^{*}, \mathbb{R}\right)$,

$$
D F(\mu) \cdot X_{H}(\mu)=\{\{F, H\}\}
$$

The left side is $\dot{F}=d F / d t$, the rate of change of $F$ under the vector field $X_{H}$. We call $\dot{F}=\{\{F, H\}\}$ the evolution equations determined by $H$ in Poisson-bracket form, while $\dot{\mu}=X_{H}(\mu)$ are the KAKS evolution equations themselves. They are clearly equivalent descriptions.

The above development may seem pretty far removed from the classical Poisson bracket

$$
\{f, g\}=\sum_{i=1}^{n}\left(\frac{\partial f}{\partial q^{i}} \frac{\partial g}{\partial p_{i}}-\frac{\partial f}{\partial p_{i}} \frac{\partial g}{\partial q^{i}}\right)
$$

and Hamilton equations

$$
\dot{q}^{i}=\frac{\partial H}{\partial p_{i}}, \quad \dot{p}^{i}=-\frac{\partial H}{\partial q^{i}} .
$$

In fact, through reduction we can derive the KAKS Poisson structure $\{\{\}$,$\} from$ the traditional Poisson brackets in a natural way. This can proceed via two equivalent routes:

Roure 1. Regard (3* as $T_{c}^{*} G$, the dual of the tangent space to $G$ at the identity $e \in G$. For $F, G: \mathbb{S}^{*} \rightarrow \mathbb{R}$, extend $F$ and $G$ to functions $f, g: T^{*} G \rightarrow \mathbb{R}$ by left translation. Then $\{f, g\}$ is defined by the classical formula where $\left(q^{i}, p_{i}\right)$ are cotangent bundle coordinates. Let $\{\{F, G\}\}$ be $\{f, g\}$ restricted to $\mathcal{B}^{*}$. Then this yields the KAKS bracket.

Route 2. $G$ acts on $\mathbb{S}^{*}$ by the co-adjoint action; i.e. the dual of the adjoint action. The latter is the linearization at the identity of the action of $G$ on $G$ by conjugation: $h \leftrightarrow g h g^{-1}$. The orbits of the coadjoint action are always symplectic manifolds. (This can be seen directly or by reducing $T^{*} G$ by the action of $G$ by left translation). Define $\{\{F, G\}\}$ at $\mu$ by restricting $F$ and $G$ to $\mathcal{O}_{\mu}$, the orbit of $\mu$ and then computing the symplectic Poisson bracket and evaluating at $\mu$. Again this gives the same KAKS bracket.

We note that $\mathcal{H}^{*}$ itself need not be a symplectic manifold (it may be odd-dimensional) but it is foliated by symplectic manifolds (route 2) and is closely associated to the symplectic manifold $T^{*} G$ (route 1 ).

An important property of KAKS systems is that they leave the coadjoint orbits invariant. This is implicit in route 2 above, but may also be checked directly. This fact is related to a conservation law in the sense of Noether reflecting the $G$-symmetry of the problem. 
If $G$ has a biinvariant inner product $\langle$,$\rangle then the KAKS equations can be$ given a little more explicitly. In this case we can identify $\mathbb{B}$ and $\mathscr{H}^{*}$ via the Killing form $($,$) and the evolution equations on \mathbb{H}^{*}$ become evolution equations on (B. (It is not always advantageous to make this identification). Denoting elements of $(\xi)$ by $\xi, \delta H / \delta \xi$ becomes $\nabla_{\xi} H$, the $\xi$-gradient of $H$, a vector field on ( 3 and the KAKS equations are easily seen to be equivalent to the following equations on $\mathfrak{H}$ :

$$
\dot{\xi}=\left[\xi, \nabla_{\xi} H\right]
$$

Example 1 (The Rigid Body). Here we take $G=S O(3)$ so that $(5$, its Lie algebra, is identifiable with $\mathbb{R}^{3}$ and the Lie bracket with the cross product. $A$ point $m \in$ (S) $^{*}$ represents the angular momentum in "body coordinates". (See Abraham and Marsden [1978] or Arnold [1978] for the explanation of this terminology). For the moment we do not identify $\mathcal{G}^{*}$ and $\mathcal{S}^{*}$. The energy $H$ is the kinetic energy of the body, a positive definite quadratic function of $m$. By choosing an appropriate orthonormal basis of $\mathbb{R}^{3}$ (and corresponding orthonormal dual basis of $\mathbb{R}^{3 *}$ ) we can assume $H$ is diagonal:

$$
H(m)=\frac{1}{2}\left(\frac{m_{1}^{2}}{I_{1}}+\frac{m_{2}^{2}}{I_{2}}+\frac{m_{3}^{2}}{I_{3}}\right)
$$

where $I_{1}, I_{2}, I_{3}$ are positive constants, the moments of inertia. Let us work out the KAKS equations $\dot{F}=\{\{F, H\}\}$ in this case. Clearly $\delta F / \delta m$ is just the vector in $\mathbb{R}^{3}$ with components $\left(\partial F / \partial m_{1}, \partial F / \partial m_{2}, \partial F / \partial m_{3}\right)$. Thus

$$
\{\{F, H\}\}(m)=-\left\langle m, \frac{\delta F}{\delta m} \times \frac{\delta H}{\delta m}\right\rangle
$$

the triple product. Choosing $F(m)=m_{1}$, the equation $\dot{F}=\{\{F, H\}\}$ reads

$$
\dot{m}_{1}=-\left|\begin{array}{ccc}
m_{1} & m_{2} & m_{3} \\
1 & 0 & 0 \\
\frac{m_{1}}{I_{1}} & \frac{m_{2}}{I_{2}} & \frac{m_{3}}{I_{3}}
\end{array}\right|=\frac{I_{2}-I_{3}}{I_{2} I_{3}} m_{2} m_{3}
$$

The equations for $\dot{m}_{2}$ and $\dot{m}_{3}$ are obtained by cyclic permutation. These are the famous Euler equations for a force-free rigid body. It is trivial to check that $(d / d t)\left(m_{1}^{2}+m_{2}^{2}+m_{3}^{2}\right)=0$ i.e. $\|m\|^{2}$ is constant in time. The spheres $\|m\|=$ constant are exactly the coadjoint orbits for $S O(3)$. Thus $S O(3)^{*}$ is the union of these symplectic manifolds (plus the origin). Their preservation by the Euler equations corresponds to the conservation of angular momentum. There is a Killing form in the case, namely the standard inner product and $\dot{\xi}=\left[\xi, \nabla_{\xi} H\right]$ yields the same Euler equations. 
Example 2 (The Heavy Top). The equations for a heavy top can also be written in KAKS form for the Euclidean group. Here $E(3)=S O(3) \times \mathbb{R}^{3}$, the semi-direct product of rotations and translations. Writing elements of the Lie algebra as paris $(m, v)$, the Hamiltonian is

$$
H=\frac{1}{2}\left(\frac{m_{1}^{2}}{I_{1}}+\frac{m_{2}^{2}}{I_{2}}+\frac{m_{3}^{2}}{I_{3}}\right)+m g v_{3}
$$

One can easily check that the KAKS equations yield the correct equations for a heavy top (see Guillemin and Sternberg [1980] and Ratiu (1981]). In case $I_{1}=I_{2}$ one has the Lagrange or symmetric top which has an additional $S^{1}$ symmetry. Using these geometric ideas, Holmes and Marsden [1981] showed that for certain $I_{1} \neq I_{2}$, the dynamics of the heavy top is chaotic; i.e. contains irregular aperiodic orbits whose closure is a complex invariant set. Here is a good example where traditional methods of classical applied mathematics need geometric augmentation in the spirit of Poincaré.

We refer to Arnold [1966], Abraham and Marsden [1978], Marsden, Ebin and Fischer [1972] and Marsden [1981] for the indication of how to similarly treat systems 3-6. In $\$ 3$ we shall discuss 7 and 10 . We now indicate how to treat 8 and 9 as they are amusing and perhaps not so well known.

ExAMPLE 8 (Quantum Mechanics). Here $\mathscr{Q}(\mathscr{H})$ is the unitary group of a complex Hilbert space $\mathscr{K}$. The Schrodinger equation

$$
i \dot{\psi}=H_{\mathrm{op}} \psi
$$

where $H_{\text {op }}$ is a self adjoint operator is well-known to be Hamiltonian relative to the symplectic form $\omega(\psi, \phi)=-\operatorname{Im}\langle\psi, \phi)$ and the energy $H(\psi)=\frac{1}{2}\left(\psi, H_{\mathrm{op}} \psi\right\rangle$ (see Marsden [1968] or Chernoff and Marsden [1974] for instance).

The Heisenberg equations, equivalent to the Schrodinger equation, are:

$$
i \dot{T}=\left[T, H_{\mathrm{op}}\right]
$$

where $T$ is a self-adjoint operator and [,] is the commutator bracket. These are in KAKS form. Indeed, the Lie algebra $u$ of $\mathcal{U}(\mathscr{H})$ consists of the skew adjoint operators; via the Hermitian inner product $\langle A, B\rangle=\operatorname{trace}\left(A B^{*}\right)$ we identify 4 and $\iota^{*}$. The Hamiltonian is $H(A)=\left\langle i H_{o p}, A\right\rangle$, which is real and the KAKS bracket is

$$
\begin{aligned}
\{\{F, H\}\}(A) & =-\left\langle A,\left[\frac{\delta F}{\delta A}, i H_{\mathrm{op}}\right]\right\rangle \\
& =\left\langle\frac{\delta F}{\delta A},\left[i H_{\mathrm{op}}, A\right]\right\rangle
\end{aligned}
$$

Thus, either directly or by our general remarks, the KAKS equations are 
equivalent to

$$
\dot{A}=i\left[H_{\mathrm{op}}, A\right]
$$

But these are the Heisenberg equations with $A=i T$. The coadjoint orbits are

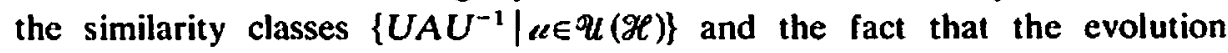
preserves these orbits is of course standard. (If we choose $A$ to be $i \times$ projection onto a 1-dimensional subspace, its coadjoint orbit is just projective Hilbert space; the other orbits are also interesting symplectic manifolds).

EXAMPLE 9. One can regard many Lax type equations that occur in nonlinear wave equations (Lax [1968]) as KAKS equations in a similar way. (For the specific example of the Cologero system, which can be dealt with by the more general reduction methods, see Marsden [1981, p. 40f] and for the KdV equation, see Abraham and Marsden [1978, §5.5])

Let $\mathscr{H}$ be a real or complex Hilbert space (finite or infinite dimensional), $\mathcal{U}$ the unitary group and a its Lie algebra, the skew Hermitian matrices. Let $L: « \rightarrow «$ be a given (non-linear) operator and consider the "Lax equation"

$$
\dot{\lambda}=[L(\lambda), \lambda]
$$

for $\lambda \in \lll$. This equation means that $\lambda$ evolves on a coadjoint orbit in $\approx \approx \varkappa^{*}$. Thus

$$
\lambda(t)=U(t) \lambda(0) U(t)^{-1}
$$

for a unitary operator $U(t)$, so $\lambda(0)$ and $\lambda(t)$ are unitarily equivalent (i.e. on the same coadjoint orbit) and so the evolution is isospectral. It is easily checked, as in the previous example, that the equations $\dot{\lambda}=[L(\lambda), \lambda]$ are in KAKS form if and only if

$$
L(\lambda)=\frac{\delta H}{\delta \lambda}
$$

for some function $H: \leadsto \rightarrow \mathbb{R}$ (with $«$ and $\varkappa^{*}$ identified by the Killing form $\langle A, B\rangle=\operatorname{trace}\left(A B^{*}\right)$.) Thus the equations are Hamiltonian as a KAKS system or in the standard sense on each coadjoint orbit in 4 .

§3. The Poisson-Vlasov equation as a KAKS system. The Poisson-Vlasov equation describes the evolution of a collisionless ionized plasma moving under the influence of self-induced electrostatic forces. When the electromagnetic field is dynamic, things are more interesting and these Maxwell-Vlasov equations are discussed later. The Poisson-Vlasov equation for a plasma moving in $\mathbb{R}^{3}$ is

$$
\frac{\partial f}{\partial t}+\sum_{i=1}^{3}\left(v^{i} \cdot \frac{\partial f}{\partial x^{i}}-\frac{1}{m} \frac{\partial \phi_{f}}{\partial x^{i}} \frac{\partial f}{\partial v^{i}}\right)=0
$$


where $\phi_{f}$ is defined by Poisson's equation:

$$
\Delta \phi_{f}=-\rho_{f}
$$

and $\rho_{f}$ is the charge determined by $f$ :

$$
\rho_{f}(x, t)=e \int f(x, v, t) d v
$$

Here $f(x, v, t)$ represents the plasma density in position-velocity space at time $t ; e$ is the ion charge and $m$ the ion mass. We are considering the motion of a cloud of charged ions of a single species for simplicity. The generalization to several species is routine. For $e=0$ we get Liouville's equation for free particles in $\mathbb{R}^{3}$.

The Hamiltonian for the Poisson-Vlasov equation is

$$
H(f)=\frac{m}{2} \int\|v\|^{2} f(x, v, t) d x d v+\frac{1}{2} \int\left\|\nabla \phi_{f}(x)\right\|^{2} d x
$$

The Poisson-Vlasov equations can be recast in bracket form as

where

$$
\dot{F}=\{\{F, H\}\}
$$

$$
\{\{F, G\}\}(f)=\int f\left\{\frac{\delta F}{\delta f}, \frac{\delta G}{\delta f}\right\} d x d v
$$

and $\{$,$\} is the standard Poisson bracket for functions of x, v$ and $\delta F / \delta f$ here coincides with the functional derivative as used in physics and the calculus of variations.

The bracket form of the Poisson-Vlasov equations can be checked directly but is unsatisfactory unless we understand how this bracket is related to symplectic geometry. We shall now show that in fact the bracket is a KAKS bracket for a Lie group. In what follows we choose natural units in which $e=m=1$. Moreover, we shall identify velocity with momentum; hence we let $\mathbb{R}^{6}$ denote the usual position-momentum phase space with coordinates $\left(x^{1}, x^{2}, x^{3}, p_{1}, p_{2}, p_{3}\right)$ and the standard symplectic structure $\omega=\sum d x^{i} \wedge d p_{i}$. Let $\mathscr{S}$ denote the group of canonical transformations of $\mathbb{R}^{6}$ (which satisfy certain growth conditions at infinity). The Lie algebra of $\mathscr{S}$ consists of the Hamiltonian vector fields on $\mathbb{R}^{6}$ (again with certain growth conditions). We shall identify elements of $\triangle$ with their generating functions, so that $\triangle$ consists of the $C^{\infty}$ functions on $\mathbb{R}^{6}$ and the (right) Lie algebra structure is given by $[f, g]=$ $-\{f, g\}$, the negative of the usual Poisson bracket on phase space. (This follows from Exercise 4.1G and Corollary 3.3.18 of Abraham and Marsden [1978]).

The dual space $\sigma^{*}$ can be identified with the distribution densities on $\mathbb{R}^{6}$ (which satisfy certain decay conditions at infinity): the pairing between $h \in 0$ 
and $f \in \sigma^{*}$ is given by integration

$$
\langle h, f\rangle=\int h f d x d p
$$

(The "density" is really $f d x d p$, but we denote it simply by f.) Now as for any Lie algebra, the dual space $\diamond^{*}$ carries a natural Poisson structure.

The general formula $\{\{F, G\}\}(\mu)=-\left\langle\mu,\left[\frac{\delta F}{\delta \mu}, \frac{\delta G}{\delta \mu}\right]\right\rangle$ becomes, in this case,

$$
\{\{F, G\}\}(f)=\int f\left\{\frac{\delta F}{\delta f}, \frac{\delta G}{\delta f}\right\} d x d p
$$

which is the bracket mentioned above. Thus indeed the Poisson-Vlasov equations can be put exactly into KAKS form.

The preservation of coadjoint orbits is equivalent to the fact that $f$ at time $t$ is related to $f$ at time $t=0$ by composition with some canonical transformation. This is an important property of the Poisson-Vlasov equation. It can also be seen by writing the equation as a non-linear self-consistent Liouville system:

$$
\frac{\partial f}{\partial t}=-\{f, \mathscr{H}(f)\}
$$

where $\{$,$\} is the ordinary Poisson bracket in x p$ space and

$$
\mathscr{H}(f)=\frac{1}{2}\|v\|^{2}+\phi_{f}(x)
$$

\$4. Maxwell's equations. Before coupling the Vlasov equation to the electromagnetic field equations, we shall consider separately the Hamiltonian description of Maxwell's equations. The appropriate Poisson bracket for the electric (E) and magnetic (B) fields will be constructed by reduction (Marsden and Weinstein [1974]).

As the configuration space for Maxwell's equations, we take the space $\mathfrak{U}$ of vector fields $A$ on $\mathbb{R}^{3}$. (These are the "vector potentials. In more general situations, one should replace $\mathscr{A}$ by the set of connections on a principal bundle over configuration space.) The corresponding phase space is then the cotangent bundle $T^{*} \mathscr{A}$. Elements of $T^{* \mathfrak{A}}$ may be identified with pairs $(A, Y)$, where $Y$ is a vector field density on $\mathbb{R}^{3}$. (As usual, we do not distinguish $Y$ and $Y d x$.) The pairing between $A$ 's and $Y$ 's is given by integration, so that the canonical symplectic structure $\omega$ on $T^{*} \mathfrak{U}$ is given by

$$
\omega\left(\left(A_{1}, Y_{1}\right),\left(A_{2}, Y_{2}\right)\right)=\int_{M}\left(Y_{2} \cdot A_{1}-Y_{1} \cdot A_{2}\right) d x
$$

with associated Poisson bracket

$$
\{F, G\}=\int\left(\frac{\delta F}{\delta A} \frac{\delta G}{\delta Y}-\frac{\delta F}{\delta Y} \frac{\delta G}{\delta A}\right) d x
$$


With the Hamiltonian

$$
H(A, Y)=\frac{1}{2} \int|Y|^{2} d x+\frac{1}{2} \int|\operatorname{curl} A|^{2} d x
$$

Hamilton's equations are easily computed to be

$$
\frac{\partial Y}{\partial t}=-\operatorname{curl} \operatorname{curl} A \text { and } \frac{\partial A}{\partial t}=Y
$$

If we write $B$ for curl $A$ and $E$ for $-Y$, the Hamiltonian becomes the usual field energy

$$
\frac{1}{2} \int|E|^{2} d x+\frac{1}{2} \int|B|^{2} d x
$$

and so Hamilton's equations imply Maxwell's equations:

$$
\frac{\partial E}{\partial t}=\operatorname{curl} B \text { and } \frac{\partial B}{\partial t}=-\operatorname{curl} E
$$

The remaining two Maxwell equations will appear as a consequence of gauge invariance. The gauge group $\mathscr{G}$ consists of real valued functions on $\mathbb{R}^{3}$; the group operation is addition. An element $\psi \in G$ acts on $\mathfrak{A}$ by the rule ${ }^{(1)}$

$$
A \mapsto A+\nabla \psi \text {. }
$$

This "translation" of $A$ extends in the usual way to a canonical transformation ("extended point transformation") of $T^{*} \mathfrak{A}$ given by

$$
(A, Y) \mapsto(A+\nabla \psi, Y) \text {. }
$$

Notice that the Hamiltonian $H(A, Y)$ is invariant under these transformations. This means that we can use the gauge symmetries to reduce the degrees of freedom of our system. The action of $\mathscr{G}$ on $T^{*} \mathfrak{A}$ has a corresponding conserved quantity, namely a map $J: T^{*} \mathfrak{U} \rightarrow \mathbb{S}^{*}$ where $\mathfrak{G}$, the Lie algebra of $\mathscr{G}$, is identified with the real valued functions on $\mathbb{R}^{3}$. The map $J$, called a momentum map, may be determined by a standard formula (Abraham and Marsden [1978, Corollary 4.2.11]): for $\phi \in \mathbb{B}$,

$$
(J(A, Y), \phi)=\int(Y \cdot \nabla \phi) d x=-\int(\operatorname{div} Y) \phi d x
$$

Thus we may write

$$
J(A, Y)=-\operatorname{div} Y
$$

\footnotetext{
(1) Notice that we work directly with three dimensional fields. Four dimensionally, one has an extra "degree" of gauge freedom associated with the time derivative $\partial_{1} \phi$. We have already eliminated this freedom and the corresponding non-dynamical field $A_{4}$ (whose conjugate momentum vanishes). This is the standard Dirac procedure for a relativistic field theory such as Maxwell's equations.
} 
If $\rho$ is an element of (G* (i.e. $\rho$ is a density on $\left.\mathbb{R}^{3}\right), J^{-1}(\rho)=$ $\left\{(A, Y) \in T^{*} \mathfrak{A} \mid \operatorname{div} Y=-\rho\right\}$. In terms of $E$, the condition div $Y=-\rho$ becomes the Maxwell equation $\operatorname{div} E=\rho$, so we may interpret the elements of $\mathscr{Q}^{*}$ as charge densities.

By a general theorem on reduction (Marsden and Weinstein (1974), the manifold $J^{-1}(\rho) / G$ has a naturally induced symplectic structure.

A little computation shows that the reduced manifold $J^{-1}(\rho) / 9$ can be identified with $\operatorname{Max}=\{(E, B) \mid \operatorname{div} E=\rho, \operatorname{div} B=0\}$, and that the Poisson bracket induced on Max is given in terms of $E$ and $B$ by

$$
\{F, G\}=\int\left(\frac{\delta F}{\delta E} \operatorname{curl} \frac{\delta G}{\delta B}-\frac{\delta G}{\delta E} \operatorname{curl} \frac{\delta F}{\delta B}\right) d x
$$

Maxwell's equations with an ambient charge density $\rho$ are thus Hamilton's equations for

$$
H(E, B)=\frac{1}{2} \int\left(|E|^{2}+|B|^{2}\right) d x
$$

on the space Max, and can be written

$$
\dot{F}=\{F, H\} \text {. }
$$

§5. The Maxwell-Vlasov equations. The Maxwell-Vlasov equations are:

$$
\begin{gathered}
\frac{\partial f}{\partial t}+v \cdot \frac{\partial f}{\partial x}+\frac{e}{m}\left(E+\frac{v \times B}{c}\right) \cdot \frac{\partial f}{\partial v}=0 \\
\frac{1}{c} \frac{\partial B}{\partial t}=-\operatorname{curl} E \\
\frac{1}{c} \frac{\partial E}{\partial t}=\operatorname{curl} B-\frac{e}{c} \int v f(x, v, t) d v \\
\operatorname{div} E=\rho_{f}, \quad \text { where } \rho_{f}=e \int f(x, v, t) d v \\
\operatorname{div} B=0
\end{gathered}
$$

(Letting $c \rightarrow \infty$ leads to the Poisson-Vlasov equation).

The Hamiltonian for the Maxwell-Vlasov system is

$$
H(f, E, B)=\int \frac{1}{2}|v|^{2} f(x, v, t) d x d v+\int \frac{1}{2}\left[|E(x, t)|^{2}+|B(x, t)|^{2}\right] d x
$$

Our goal is to understand the Hamiltonian structure of these equations. (As usual, we let $e=m=c=1$ ).

The Hamiltonian structure for the Maxwell-Vlasov system is very simple if we choose as our variables densities on $(x, p)$ space (rather than $(x, v)$ space) 
and elements $(A, Y)$ of $T^{*} \mathscr{A}$. To avoid confusion with densities $f$ on $(x, v)$ space, we shall use the notation $f_{\text {mom }}$ for densities on $(x, p)$ space.

The Poisson structure on $s^{*} \times T^{*} \mathfrak{A}$ is just the sum of those on $s^{*}$ and $T^{*} \mathfrak{U}$ : for functions $\bar{F}$ and $\bar{G}$ of $f_{\text {mom }}, A$, and $Y$, set

$$
\{\{\bar{F}, \bar{G}\}\}\left(f_{\mathrm{mom}}, A, Y\right)=\int f_{\mathrm{mom}}\left\{\frac{\partial \bar{F}}{\delta f_{\mathrm{mom}}}, \frac{\delta \bar{G}}{\delta f_{\mathrm{mom}}}\right\} d x d p+\int\left(\frac{\delta \bar{F}}{\delta A} \frac{\delta \bar{G}}{\delta \mathrm{Y}}-\frac{\delta \bar{G}}{\delta A} \frac{\delta \bar{F}}{\delta Y}\right) d x
$$

and the Hamiltonian is just $H(f, E, B)$ written in terms of these variables. Using the classical relation $p=v+A$ between momentum and velocity we get

$$
\bar{H}\left(f_{\mathrm{mom}}, A, Y\right)=\frac{1}{2} \int|p-A(x)|^{2} f_{\text {mom }}(x, p) d x d p+\frac{1}{2} \int\left(|Y|^{2}+|\operatorname{curl} A|^{2}\right) d x
$$

One now computes easily that the evolution equations $\bar{F}=\{\{\bar{F}, \bar{H}\}\}$ for a function $\bar{F}$ on $\partial^{*} \times T^{*} \mathscr{U}$ are the Maxwell-Vlasov equations with $\frac{\partial B}{\partial t}=-\operatorname{curl} E$ replaced by $\frac{\partial A}{\partial t}=Y$. To get the Maxwell-Vlasov equations as written we must, as in the case of Maxwell's equations, reduce by the action of $\mathscr{G}$.

The natural action of $\mathscr{G}$ on $\sigma^{*}$ is defined by letting $\psi \in \mathscr{G}$ act by the (linear) map

$$
f_{\text {mom }} \mapsto f_{\text {mom }}{ }^{\circ} \tau_{-\nabla \psi}
$$

where $\tau_{-\nabla_{\psi}}: \mathbb{R}^{6} \rightarrow \mathbb{R}^{6}$ is the "momentum translation map" defined by

$$
\tau_{-\nabla \psi}(x, p)=(x, p-\nabla \psi(x)) .
$$

It is easy to verify that $\tau_{-v_{\psi}}$ is a canonical transformation, so it preserves the ordinary Poisson bracket on $\mathbb{R}^{6}$. It follows that this action preserves the Poisson structure on $a^{*}$. A simple calculation shows that it has a momentum map $J: \delta^{*} \rightarrow$ (B) $^{*}$ given by $J\left(f_{\text {mom }}\right)=-\int f_{\text {mom }}(x, p) d p$.

Now we define the action of $\mathscr{G}$ on the product $a^{*} \times T^{*} \mathfrak{U}$ as follows: $\psi \in \mathscr{G}$ maps

$$
\left(f_{\text {mom }}, A, Y\right) \mapsto\left(f_{\text {mom }}{ }^{\circ} \tau_{-\nabla \psi}, A+\nabla \psi, Y\right) .
$$

This action leaves the Hamiltonian $\bar{H}$ invariant.

The momentum map $J: 0^{*} \times T^{*} \mathfrak{U}\left(J^{*}\right.$ for this action is given by:

$$
J\left(f_{\text {mom }}, A, Y\right)=-\int f_{\text {mom }}(x, p) d p-\operatorname{div} Y .
$$

With this action we are ready to consider the reduced manifold $\left(s^{*} \times T^{*} \mathfrak{A}\right)_{0}=$ $J^{-1}(0) / 9$. This space may be identified with the Maxwell-Vlasov phase space

$$
\mathscr{M V}=\left\{(f, B, E) \mid \operatorname{div} B=0 \text { and } \operatorname{div} E=\int f(x, v) d v\right\}
$$


by associating to each $\left(f_{\mathrm{mom}}, A, Y\right)$ in $J^{-1}(0)$ the triple $(f, B, E)$ in $M V$ where

$$
f(x, v)=f_{\text {mom }}(x, v+A(x)), B=\operatorname{curl} A, \text { and } E=-Y .
$$

By the general theory of reduction, $\mu V$ inherits a Poisson structure from the one on $\delta^{*} \times T^{*} \mathscr{U}$. Since the Hamiltonian $(\bar{H})$ is invariant under $\mathscr{G}$, it follows that the Maxwell-Vlasov equations are a Hamiltonian system on $\mathcal{M V}$ with respect to this structure. One can, in fact with a little labor compute the explicit form of the inherited Poisson structure in the variables $(f, B, E)$. It is given by the (perhaps unexpectedly complex) formula

$$
\begin{aligned}
\{\{F, G\}\}(f, E, B)= & \int f\left\{\frac{\delta F}{\delta f}, \frac{\delta G}{\delta f}\right\} d x d v+\int\left(\frac{\delta F}{\delta E} \operatorname{curl} \frac{\delta G}{\delta B}-\frac{\delta G}{\delta E} \operatorname{curl} \frac{\delta F}{\delta B}\right) d x \\
& +\int\left(\frac{\delta F}{\delta E} \cdot \frac{\partial f}{\partial v} \frac{\delta G}{\delta f}-\frac{\delta G}{\delta E} \cdot \frac{\partial f}{\partial v} \frac{\delta F}{\delta f}\right) d x d v \\
& +\int f B \cdot\left(\frac{\partial}{\partial v} \frac{\delta F}{\delta f} \times \frac{\partial}{\partial v} \frac{\delta G}{\delta f}\right) d x d v
\end{aligned}
$$

The Maxwell-Vlasov equations can thus be written as

$$
\dot{F}=\{\{F, H\}\}
$$

We know by general principles that the bracket $\{\{\}$,$\} must satisfy Jacobi's$ identity. Indeed we obtained it by totally natural constructions. To verify Jacobi's identity by hand would be extremely tedious. In fact a first attempt at producing such a bracket was done by hand using brilliant guesswork by Morrison [1980]. However his bracket fails to satisfy Jacobi's identity (Weinstein and Morrison [1981]).

Having a Hamiltonian structure for the Maxwell-Vlasov equations is of great interest to plasma physicists because

(a) it enables them to begin to make use of powerful techniques of perturbation theory for Hamiltonian systems

(b) the relationship between the classical, quantum and semiclassical theories can be attacked in a useful context.

(c) the relationship between the full theory and various Hamiltonian truncations (such as the three wave interaction model) should now be understandable.

Similar Hamiltonian structures to these that we have found for plasmas can also be given for the equations of magnetohydrodynamics; cf. Morrison and Greene [1980].

Hopefully the situation described above reinforces the fact that current geometric methods in mechanics do have a role to play in concrete physical problems. 


\title{
REFERENCES
}

R. Abraham and J. Marsden [1978]. Foundations of Mechanics, Second Edition, W. A. Benjamin Co.

V. Arnold [1966]. Sur la geometrié differentielle des groupes de Lie de dimension infinite et ses applications a l'hydrodynamique des fluids parfaits, Ann. Inst. Grenoble 16, 319-361.

V. Arnold [1968]. Mathematical methods of classical mechanics. Springer Graduate Texts in Math. No. 60 Springer-Verlag, New York.

P. Chernoft and J. Marsden [1974]. Properties of Infinite Dimensional Hamiltonian Systems, Springer Lecture Notes \#425.

A. J. Chorin, T. J. R. Hughes, M. F. MeCracken and J. E. Marsden [1978]. Product formulas and numerical algorithms, Comm. Pure and Appl. Marh. 31, 205-256.

D. Ebin and J. Marsden [1970]. Groups of diffeomorphisms and the motion of an incompressible fluid, Ann. of Math. 92, 102-163.

V. Guillemin and S. Sternberg [1980]. The moment map and collective motion. Ann. of Phys. 127, 220-253.

P. J. Holmes and J. E. Marsden [1981]. Horseshoes and Arnold Diffusion for Hamiltonian systems on Lie Groups (to appear).

P. D. Lax [1968]. Integrals of nonlinear equations of evolution and solitary waves, Comm. Pure and Appl. Math. 21, 467-490.

J. E. Marsden [1968]. Hamiltonain One-Parameter Groups, Anch. Rat. Mech. An. 28, 362-396.

J. E. Marsden [1981]. Lectures on geometric methods in mathematical physics, CBMS-NSF Conference Series \#37, SIAM.

J. E. Marsden, D. G. Ebin and A. E. Fischer [1972]. Diffeomorphism Groups, Hydrodynamics and General Relativity, 13th Biennial Seminar of Can. Math. Congress (J. Vanstone, ed.) 135-279.

J. E. Marsden and A. Weinstein [1974]. Reduction of symplectic manifolds with symmetry, Reports on Math. Phys. 5, 121-130.

J. Marsden and A. Weinstein [1982]. The Hamiltonian Structure of the Maxwelf-Vlasov equations, Physica $D$ (to appear).

P. J. Morrison [1980]. The Maxwell-Vlasov equations as a continuous Hamiltonian system, Phys. Lett. 80A, 383-386.

P. J. Morrison and J. M. Greene [1980]. Noncanonical Hamiltonian density formulation of hydrodynamics and ideal magnetohydrodynamics, phys. Reu. Letters. 45, 790-794.

$T$. Ratju [1981]. Euler-Poisson equations on Lie algebras and the $\boldsymbol{N}$-dimensional heavy rigid body, Proc. Nall. Acad. Sci. U.S.A. 78, 1327-1328.

A. Weinstein and P. Morrison [1981]. Comments on: The Maxwell-Vlasov equations as a continuous Hamiltonian system. Phys. Lett. 86A, 235-236.

\author{
Departaent of Mathematics \\ UNJVERSTY OF CALIFORNIA \\ Berkeley, California 94720
}

